\title{
Prediction of favourable sites for spin labelling of proteins
}

\author{
Yevhen Polyhach and Gunnar Jeschke* \\ Laboratory of Physical Chemistry, ETH Zurich, Zurich, Switzerland
}

\begin{abstract}
Electron paramagnetic resonance studies on diamagnetic proteins are based on site-directed spin labelling and require relatively much effort for engineering cystein point mutations as well as expressing and labelling the protein. Therefore, it is advantageous to predict those sites and pairs of sites that can provide the most precise and most reliable information on accessibility and distances. Systematic site scans based on a rotamer library approach for spin label side groups allow for such predictions. Figures of merit are defined that can be used to rank sites according to their potential suitability in studies of structure and structural changes. Such site scans can still provide useful results if only a backbone model and the amino acid sequence of the protein are known.
\end{abstract}

Keywords: EPR spectroscopy, site-directed spin labelling, membrane proteins, molecular modelling, distance measurements

\section{Introduction}

Site-directed spin labelling (SDSL) of proteins [12], has recently become a widespread technique, in particular, in combination with pulsed electron paramagnetic resonance (EPR) techniques for the measurement of distance distributions between two spin labels $[13,17,20]$. It was early recognized that the separation between the electron spin and the protein backbone poses problems in precise modelling of structures and structural changes [3]. A more detailed study based on a molecular dynamics (MD) approach demonstrated that conformational distribution of the spin label can provide the major contribution to the width of the measured distance distributions [16]. To model this conformational distribution and to predict spin-to-spin distance distributions from a given model for the protein structure, we have developed a rotamer library approach [13] that is similar in spirit to an approach for understanding spin label dynamics $[18,19]$.

This approach has so far yielded a structural model of the functional dimer of the $\mathrm{Na}^{+} / \mathrm{H}^{+}$antiporter NhaA of E. coli [11], which was later confirmed by a mutation and crosslinking study [9] and by electron crystallography [2]. Furthermore, a coarse-grained model was obtained for a kinked transmembrane helix in the proline/ $\mathrm{Na}^{+}$symporter PutP of E. coli [10], whose full structure remains unknown. In cases of known structures, satisfying agreement was found between measured distance distributions and distance distributions predicted from crystal structures by the rotamer library approach [6,8]. A more sophisticated approach to rotamer library generation and computation of the interaction between spin label and protein has been developed and will be described in detail elsewhere.

\footnotetext{
${ }^{*}$ Corresponding author: Gunnar Jeschke, Laboratory of Physical Chemistry, ETH Zurich, Wolfgang-Pauli-Strasse 10, CH8093 Zurich, Switzerland. Tel.: +41 44632 5702; Fax: +41 44633 1448; E-mail: gunnar.jeschke@ phys.chem.ethz.ch.
} 
The new rotamer library approach allows for prediction of the conformational distribution of a spin label with a common personal computer in a computation time between less than a minute and a few minutes. This opens up the possibility to systematically scan a protein structure for favourable spin labelling sites and site pairs. Such computational site scans can potentially decrease the expense of SDSL studies and provide more reliable results. Both very tight sites, where labelling fails or leads to changes in structure and function and very loose sites, where distance distributions are very broad, can be avoided. Site scans are most useful in situations where the structure of the studied proteins is known and either structural changes $[6,8]$ or the relative arrangement of several protein molecules [11] are of interest.

In this paper we consider which information can be obtained from a computational site scan and how the sites can be ranked in terms of suitability for certain EPR experiments. In particular, we consider measurements of spin label accessibility [1] and distance measurements, as well as the study of structural changes.

\section{Information from rotamer attachment to a single site}

Our approach for modelling of the conformational distribution of spin labels is based on a library of about $N=100-200$ rotameric states whose relative energies in the absence of interaction with the protein are known approximately. To identify these states and estimate their relative energies we have performed MD simulations of the free spin label with the CHARMM27 force field, using a recently published parametrization for the methanethiosulfonate spin label (MTSL) [18] and our own parametrization for the iodoacetamido-proxyl (IA-Proxyl) spin label. Briefly, the states are derived by analyzing maxima in the population of individual dihedral angles. The internal populations $p_{i}$ of these states can be obtained by analyzing all frames of the MD trajectory in terms of the closest rotamer. These populations are related to relative energies $e_{i}$ of the unconstrained label via the Boltzmann distribution, $p_{i} / p_{\max }=\exp \left(-e_{i} / k T\right)$, where $p_{\max }$ is the population of the most populated rotamer, whose relative energy is defined as zero, $k$ is the Boltzmann constant and $T$ the simulation temperature.

The relative energies of the rotamers attached at a certain site of the protein are computed by adding to the $e_{i}$ further energies $\varepsilon_{i}$ that correspond to the interaction between label and protein. The interaction energy $\varepsilon_{i}$ for a particular rotamer is a sum of pairwise Lennard-Jones potential energies between one atom of the label and one atom of the protein. A distance cutoff is used to speed up computations and scaling of the van der Waals radii in the Lennard-Jones potentials $[4,14]$ is applied to empirically account for flexibility of the protein structure. Details of the approach and validation data will be published elsewhere.

This approach yields, for each site-attached rotamer, a predicted population $\pi_{i}=p_{i} \exp \left(-\varepsilon_{i} / k T\right) / Z$ and, for the whole set of rotamers, the partition function $Z=\sum p_{i} \exp \left(-\varepsilon_{i} / k T\right)$. The partition function $Z$ is a measure for the tightness of the site, with small values of $Z$ corresponding to large positive interaction energies between label and protein. Very small $Z$ indicate that labelling may fail or, if it succeeds, is likely to cause a change in protein structure. In predictions, we assume that for the MTS rotamer library consisting of 210 rotamers $Z<0.05$ indicates difficulties in labelling. This threshold corresponds to a situation where label attachment becomes less favourable by an average strain energy of approximately $7.5 \mathrm{~kJ} / \mathrm{mol}$ compared to an unconstrained site.

As a measure for the width of the conformational ensemble, we use the number $n$ of most populated rotamers that together make up $99.5 \%$ of the total population. This number is correlated to the partition 
function $Z$, however, the correlation is not strict. For instance, a single, low-energy rotamer can lead to a larger value $Z$ than tens of rotamers that all have high, but similar energies. These two scenarios correspond, respectively, to a favourable tight site, where the distribution is narrow and energy low, and an unfavourable tight site, where the distribution is broad and energy high.

From an experimental point of view, the spatial distribution of the midpoint of the nitroxide $\mathrm{N}-\mathrm{O}$ bond is of greater interest than the number of populated rotamers. This midpoint is the approximate site of the electron spin. A broad spatial distribution induces ambiguities in accessibility studies and may cause broad distance distributions. These questions are now discussed in turn.

\section{Site choice for accessibility measurements}

The accessibility of the spin label to water or oxygen is most easily interpreted when the spatial distribution of the label is narrow. This can be quantified by the root mean square deviation of the midpoint of the $\mathrm{N}-\mathrm{O}$ group from its mean position,

$$
\sigma_{\mathrm{NO}}=\left(0.005 \mathrm{~nm}^{2}+N /(N-1) \sum \pi_{i}\left[\left(x_{i}-\langle x\rangle\right)^{2}+\left(y_{i}-\langle y\rangle\right)^{2}+\left(z_{i}-\langle z\rangle\right)^{2}\right]\right)^{1 / 2} .
$$

In Eq. (1) $x_{i}, y_{i}$ and $z_{i}$ are the Cartesian coordinates of the $\mathrm{N}-\mathrm{O}$ midpoints of the rotamers $(I=$ $1, \ldots, N)$, while $\langle x\rangle=\sum \pi_{i} x_{i}$ is the mean value of the $x$ coordinates and $\langle y\rangle$ and $\langle z\rangle$ are defined analogously. The offset of $0.005 \mathrm{~nm}^{2}$ accounts for the spatial distribution due to nitroxide libration motion that is expected even if only one rotamer is populated [19].

For MTSL attached to an isolated cystein, i.e., for unconstrained MTSL, we find $\sigma_{\mathrm{NO}}=0.64 \mathrm{~nm}$ (see Fig. 1). Note that in special cases slightly larger values may occur for constrained MTSL. This happens if conformations that are close to the mean conformation are more strongly depopulated than conformations that are far from the mean conformation. The smaller $\sigma_{\mathrm{NO}}$, the more favourable is the site for an accessibility study, as long as the partition function $Z$ indicates that the site can be labelled without structural distortion. In the following, such favourable sites with $\sigma_{\mathrm{NO}} \leqslant 0.2 \mathrm{~nm}$ are termed well-constrained sites without collisions (WCSWC).

If the orientation of a membrane protein in a lipid bilayer is known, symmetry suggests a slightly different figure of merit. Assume without loss in generality that the bilayer normal is the $z$ axis of the coordinate frame. Spatial distribution within the $x y$ plane has smaller consequences than spatial distribution along the $z$ axis, since, to a first approximation, the concentrations of water and oxygen in the bilayer vary exclusively along $z$. Although presence of the protein itself may induce some variation in the $x y$ plane, the quantity:

$$
\sigma_{\mathrm{NO}, z}=\left((0.005 / 3) \mathrm{nm}^{2}+N /(N-1) \sum \pi_{i}\left(z_{i}-\langle z\rangle\right)^{2}\right)^{1 / 2}
$$

may be a better predictor for structure-related changes in accessibility than $\sigma_{\mathrm{NO}}$.

\section{Site pair choice for distance measurements}

For measurements of the distance $r_{\mathrm{NO}, \mathrm{NO}}$ between two spin labels, both labels must have a partition function $Z$ that indicates labelling without structural distortion. Some label pairs can be excluded without 


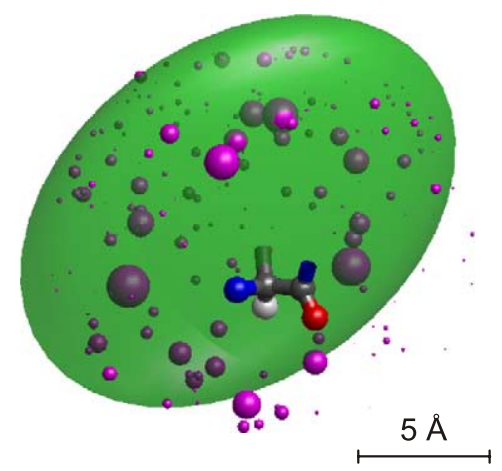

Fig. 1. Visualization of the distribution of midpoints of the N-O bond for completely unrestricted MTSL. The backbone atoms of the labelled residue, the $\mathrm{C}^{\alpha}-\mathrm{C}^{\beta}$ bond (pointing to the back) and the bond from the carbonyl $\mathrm{C}$ to the next residue (pointing to the front, slightly right) are shown as a ball and stick plot. Rotameric states are visualized as magenta spheres at the $\mathrm{N}-\mathrm{O}$ midpoints with the radius proportional to the internal population $p_{i}$. The spatial distribution of the $\mathrm{N}-\mathrm{O}$ midpoints is visualized as a transparent green ellipsoid with half axes corresponding to two times the standard deviation of the N-O midpoint coordinates. (The colors are visible in the online version of the article; http://dx.doi.org/10.3233/SPE-2010-0490.)

detailed analysis of the distribution of conformations, as they correspond to mean distances that are either too short $\left(r_{\mathrm{NO}, \mathrm{NO}}<0.8 \mathrm{~nm}\right)$ or too long $\left(r_{\mathrm{NO}, \mathrm{NO}}>7 \mathrm{~nm}\right)$. Although very short distances can be detected in principle and distance estimates can be obtained from the intensity of half-field transitions [5,7], interpretation in the presence of conformational distribution of a nitroxide label would be unreliable. Distances longer than about $7 \mathrm{~nm}$ cannot usually be measured in proteins with reasonable precision and currently available techniques [13].

The $\sigma_{\mathrm{NO}}$ values of the two labelled sites are not necessarily a good indicator for the expected width of the distance distribution. This is because the spatial distribution of the $\mathrm{N}-\mathrm{O}$ midpoints is not spherical. The origin of this effect is illustrated in Fig. 1 for MTSL attached to an isolated cystein. For this situation with an unconstrained conformational space, the inertia tensor of the $\mathrm{N}-\mathrm{O}$ midpoints, considering only the internal populations $p_{i}$, is visualized as a green semi-transparent ellipsoid. The direction of maximum spatial extent is along the principal axis of this tensor corresponding to the largest principal value of $0.44 \mathrm{~nm}^{2}$; it includes an angle of $70.2^{\circ}$ with the $\mathrm{C}^{\alpha}-\mathrm{C}^{\beta}$ bond and an angle of $27^{\circ}$ with the peptide plane. If the spin-spin vector is parallel to this direction, the width of the distance distribution is maximum. The direction of minimum spatial extent corresponds to the principal axis with the smallest principal value of $0.27 \mathrm{~nm}$; it includes an angle of $96^{\circ}$ with the $\mathrm{C}^{\alpha}-\mathrm{C}^{\beta}$ bond and an angle of $46^{\circ}$ with the peptide plane. If the spin-spin vector is parallel to this direction, the width is minimum. For completely unrestricted MTSL, the ratio of the maximum and minimum principal value of the tensor of spatial extension, corresponding to standard deviations of the $\mathrm{N}-\mathrm{O}$ midpoint coordinates, is 1.6. Note that the effect may be stronger if a subset of rotamers is selected due to interaction with the protein. For instance, if two subsets of conformations are populated, the distance distribution is most strongly broadened if the spin-spin vector is parallel to the vector between the centres of masses of the $\mathrm{N}-\mathrm{O}$ midpoints of the two subsets and it is least broadened if the spin-spin vector is perpendicular to this vector.

The value of experimental distance restraints is related to the relative width $\sigma_{\mathrm{r}} /\langle r\rangle$ of the distance distribution, where $\langle r\rangle$ is the mean distance and $\sigma_{\mathrm{r}}$ the standard deviation. This relative width is also most relevant in pulsed EPR distance measurements, as the effect of distance distribution on the shape 
of the dipolar evolution function is proportional to this parameter. Hence, we consider $\sigma_{\mathrm{r}} /\langle r\rangle$ as the figure of merit for a pair of sites. Here $\langle r\rangle$ is defined as:

$$
\langle r\rangle=\sum_{i} \sum_{k} \pi_{i} \pi_{k} r_{i j} / \sum_{i} \sum_{k} \pi_{i} \pi_{k}
$$

with indices $i$ and $k$ running over the $N$ rotamers at the first and second site, respectively, and $r_{i j}$ is the distance between the $\mathrm{N}-\mathrm{O}$ midpoints for a given combination of rotamers. Likewise, $\sigma_{\mathrm{r}}$ is defined as:

$$
\sigma_{\mathrm{r}}=\left(0.01 \mathrm{~nm}^{2}+N^{2} /\left(N^{2}-1\right) \sum_{i} \sum_{k} \pi_{i} \pi_{k}\left(r_{i j}-\langle r\rangle\right)^{2} / \sum_{i} \sum_{k} \pi_{i} \pi_{k}\right)^{1 / 2} .
$$

\section{Studying structural changes}

A particular strength of SDSL EPR is the characterization of structural differences between different states of a biological system, if the structure of only one of these states is known. In many of these cases there exists an initial hypothesis about the structural change that is to be tested. Such a hypothesis usually involves relative movements of protein domains or of the individual peptide chains in a complex. The individual domains or chains can be approximated as rigid bodies. In such cases, the hypothesis can be translated into an approximate model of the structure of the unknown state and a second site scan can be performed for this model. If there are several conflicting hypotheses for the structural change, site scans have to be performed for each corresponding model.

The figures of merit for sites and site pairs are then determined by relative changes between the states. For instance, in an accessibility study with known orientation of the system with respect to the bilayer, the mean change $\langle\Delta z\rangle$ in the $z$ coordinate of the $\mathrm{N}-\mathrm{O}$ midpoints divided by the larger of the two $\sigma_{\mathrm{NO}, z}$ values predicts the potential of the experiment to detect the change.

For distance measurements by pulsed EPR techniques, experimental form factors (dipolar evolution data after background correction) can be predicted from the simulated distance distribution. In this case, the figure of merit for a pair of sites is the root mean square deviation between the normalized form factors for the two states. The larger this deviation is, the easier it is to detect the structural change.

\section{Further considerations}

The figures of merit that result from computational site scans are not failsafe predictors of favourable and unfavourable labelling sites. This is, first, because the rotamer library approach provides only an approximate prediction of the conformational distribution and, second, because other structural aspects of the sites may influence the suitability for a certain experiment. We consider two examples of such structural aspects, one for accessibility and one for distance measurements.

If the labelled site is buried in the protein the accessibility to water and oxygen is low and accessibility changes are expected to be below the detection limit. Such sites can be recognized by inspecting the predicted favourable sites by protein and label visualization. In a future, more quantitative approach, accessibility will be predicted by using a model for water and dioxygen distribution in the bilayer and by considering explicitly that protein atoms exclude water and dioxygen. 
If EPR measurements are performed with deuterated buffers, water-exposed spin labels feature much longer transverse relaxation times $T_{2}$ than lipid- or detergent-exposed labels [21]. As the reliability and precision of measured distances increases with increasing $T_{2}$, pairs of water accessible sites with moderately broad distance distributions may be more favourable than lipid-exposed pairs with narrower distributions. Note also that one water-exposed label is sufficient to measure data with a long maximum dipolar evolution time, even if the label at the other site has a short $T_{2}$.

\section{Labelling statistics for two membrane proteins}

Computational site scans may provide estimates on the variability of the conformational distribution of spin labels in proteins and may identify motifs that lead to a well-defined position of the electron spin. Here we discuss such scans for two membrane proteins (Fig. 2), the leucine transporter LeuT $\mathrm{Aa}_{\mathrm{Aa}}$ of Aquifex aeolicus (PDB entry 2A65) [22] and the outer membrane protein A of Escherichia coli OmpA (PDB entry 1QJP) [15]. The two structures were selected because of their relatively high and comparable resolution of $1.65 \AA$. Furthermore, LeuT $_{\mathrm{Aa}}$ is a paradigm for sodium dependent secondary transporters with an $\alpha$-helical bundle structure and thus is related to PutP [10,22], while OmpA belongs to the second main class of integral membrane proteins that have $\beta$-barrel structures. We assumed a temperature of $175 \mathrm{~K}$ corresponding to solidification of a lipid bilayer environment. Detergent molecules and water molecules were removed from the structures, as it is assumed that a spin label could displace them.

The site scan of LeuT $_{\mathrm{Aa}}$ with MTSL predicts that out of the 509 residues resolved in the structure, $120(24 \%)$ correspond to tight sites where spin labelling might fail or distort structure. For further 10 residues, significant population of only one rotamer is observed, whereas the partition function is at least 0.1 (typically $>0.4$ ). A total of $52 \mathrm{WCSWC}$ (about 10\%) have $\sigma_{\mathrm{NO}} \leqslant 0.2 \mathrm{~nm}, 48$ of those are situated in helices and only 4 in loops. Of these WCSWC in loops, only one is more than 2 residues away from the next helix. The WCSWC in helices are distributed over nearly the whole structure, except for transmembrane helices TM7, TM9 and TM12 and the helical domain of IL5. A particularly high incidence of 11 WCSWC (33\% of all residues) is seen in TM8. Seven of the WCSWC are very close
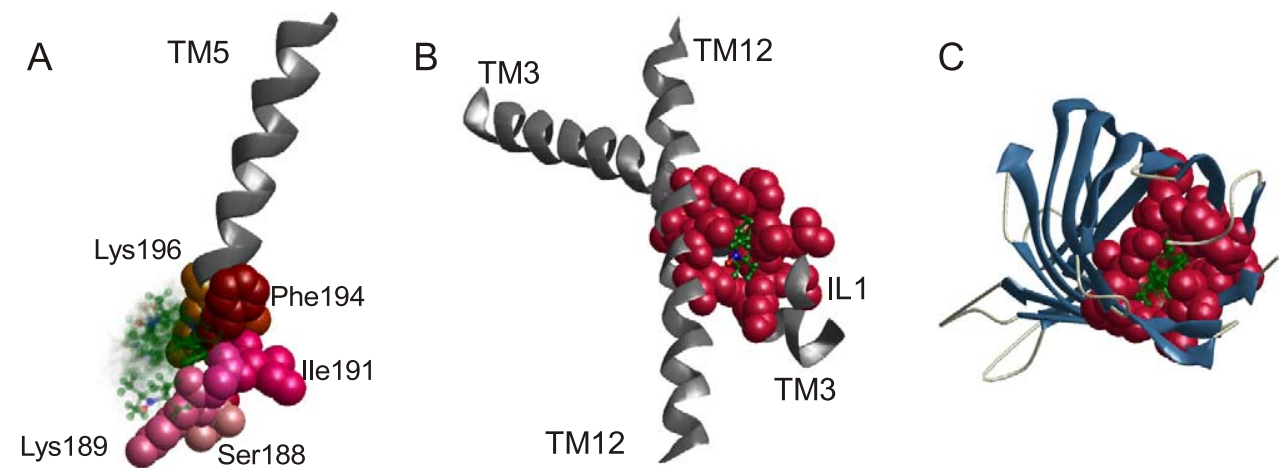

Fig. 2. Conformational distribution of MTSL constrained by side groups of other residues (rotamer library simulations). Green ball and stick plots are allowed MTSL conformations, with the transparency encoding population. The N-O nitrogen and oxygen atoms shown in blue and red, respectively. Red or reddish space-filling plots denote spatially close sidechains that

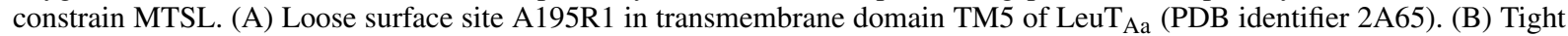
core site A95R1 in transmembrane domain TM5 of LeuT $\mathrm{Aa}_{\mathrm{Aa}}$ (C) Tight core site F40R1 in OmpA. (The colors are visible in the online version of the article; http://dx.doi.org/10.3233/SPE-2010-0490.) 
to the substrate binding site and five more are residues that are conserved in related human transporters [5]; labelling of these residues would certainly affect and possibly block transport.

For all WCSWC, without a single exception, we find that label conformations are restricted by side groups of some residues that are remote in the sequence, yet spatially close. This indicates that bulky neighbouring side groups in the context of an isolated helix are not sufficient to strongly constrain MTSL conformations. Hence, WCSWC cannot be predicted from sequence and secondary structure information alone. Furthermore, the WCSWC are almost randomly distributed among the types of original residues, except perhaps for a preference for Phe sites (11 out of 50, 22\%) and a rejection of Pro sites (1 out of $24,4 \%$ ) and Gly sites ( 2 out of $45,4 \%)$.

Among the 137 potential sites in OmpA, 17 correspond to tight sites where spin labelling might fail or distort structure $(12 \%)$. The lower incidence of such sites compared to $\mathrm{LeuT}_{\mathrm{Aa}}$ may be due to the larger fraction of surface sites in a $\beta$-barrel compared to an $\alpha$-helical bundle structure. Likewise, the incidence of WSWC is lower ( 8 WCSWC, 6\%). All WCSWC are found in strands, whereas in the whole protein 30 residues (22\%) are situated in loops. The spin-labelled side groups of all WCSWC are oriented towards the inner of the barrel. Note however that at site F123R1, where the spin label is pointing to the outside, spatial distribution of the electron spin is only slightly broader with $\sigma_{\mathrm{NO}}=0.21 \mathrm{~nm}$, although as many as 31 rotamers contribute significantly to the distribution at this site.

Consider now the case where no high-resolution structure is available. If a coarse-grained model of the backbone exists, sidechains can be attached by the SCWRL4 [14] program. We have tested this approach by performing a site scan for a decoy structure that was obtained from only the backbone coordinates of LeuT $\mathrm{Aa}_{\mathrm{Aa}}$ by attaching the side chains with SCWRL4. This test also reveals sensitivities of the rotamer library approach to differences in the conformation of side chains in the vicinity of spin labels.

When comparing the predicted mean coordinates of the $\mathrm{N}-\mathrm{O}$ midpoints in the site scans on the $\mathrm{LeuT}_{\mathrm{Aa}}$ X-ray structure and on the structure with SCWRL4 predicted side groups, we find that the deviation is smaller than $0.1 \mathrm{~nm}$ for 268 residues (53\%) and smaller than $0.2 \mathrm{~nm}$ for 364 residues (72\%). For $\sigma_{\mathrm{NO}}$ we find a deviation smaller than $0.1 \mathrm{~nm}$ for 455 residues (89\%) and smaller than $0.2 \mathrm{~nm}$ for 487 residues $(96 \%)$.

Larger deviations than $0.2 \mathrm{~nm}$ occur mainly for tight sites where significant population is predicted for only one or two rotamers. As an example we discuss residue 382 for which the maximum deviation of the mean $\mathrm{N}-\mathrm{O}$ positions of $0.62 \mathrm{~nm}$ and the $\sigma_{\mathrm{NO}}$ values deviate by $0.72 \mathrm{~nm}$ (Fig. 3). With the LeuT $\mathrm{Aa}_{\mathrm{Aa}} \mathrm{X}$-ray

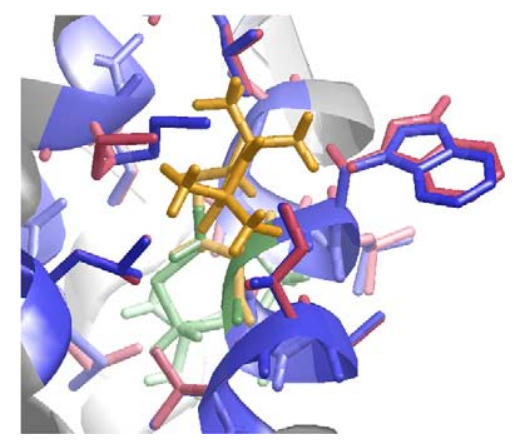

Fig. 3. Tight site (T382R1) where strongly different rotamer distributions are predicted from the X-ray structure of LeuT $\mathrm{T}_{\mathrm{Aa}}$ (blue stick plots for side groups) and from a structure where all side groups were remodelled by SCWRL4 (red stick plots). Only one MTSL rotamer (green) is significantly populated in the X-ray structure, while a second one (yellow) appears with the SCWRL4 prediction. The reason is a different conformation for residue Met 363 (arrow). (The colors are visible in the online version of the article; http://dx.doi.org/10.3233/SPE-2010-0490.) 
structure, significant population of only one rotamer, shown as a green stick plot, is predicted. With side group conformations predicted by SCWRL4, a second rotamer in a completely different position is significantly populated (yellow stick plot). This can be traced back to a different prediction for the conformation of residue Met 363 by SCWRL4 (arrow) which relieves a clash that depopulates this MTSL rotamer in the X-ray structure. It is likely that the side group of Met 363 would flip on labelling, i.e., that the prediction from the $\mathrm{X}$-ray structure underestimates the accessible conformational space for the label in this case.

\section{Conclusion}

Computational site scans based on the rotamer library approach [13] provide an approximate fast prediction of the conformational distribution of spin labels. Very tight positions, where labelling is likely to fail or distort structure, can be revealed and figures of merit of a labelling site can be defined for different EPR experiments. Such figures of merit should always be critically examined with the intended experiment in mind, using visualization of the spin label rotamers and their structural context. The assumption of fixed positions of the side groups of neighbouring residues may lead to an underestimate of conformational flexibility for tight sites. Work on an approach that allows for side group repacking is now in progress.

It is expected that systematic computational site scans before mutant engineering and chemical labelling will lead to a decrease in the expense of SDSL EPR studies and improve the information content and reliability of the results, in particular for distance measurements. To facilitate such site scans, the rotamer library and site scan approaches were implemented as modules of the Matlab-based protein visualization and modelling program MMM (Multiscale Modelling of Macromolecular Systems). This open-source program can be downloaded from http://www.epr.ethz.ch/software/index.

\section{Acknowledgement}

Financial support by Swiss National Science Foundation is gratefully acknowledged.

\section{References}

[1] C. Altenbach, D.A. Greenhalgh, H.G. Khorana and W.L. Hubbel, Proc. Natl. Acad. Sci. 91 (1994), 1667-1671.

[2] M. Appel, D. Hizlan, K.R. Vinothkumar, C. Ziegler and W. Kuhlbrandt, J. Mol. Biol. 386 (2009), 351-365.

[3] P.P. Borbat, H.S. Mchaourab and J.H. Freed, J. Am. Chem Soc. 124 (2002), 5304-5314.

[4] A.A. Canutescu, A.A. Shelenkov and R.L. Dunbrack Jr., Protein Sci. 12 (2003), 2001-2014.

[5] R.E. Coffman and A. Pezeshk, J. Magn. Reson. 70 (1986), 21-33.

[6] C. Dockter, A. Volkov, C. Bauer, Y. Polyhach, Z. Joly-Lopez, G. Jeschke and H. Paulsen, Proc. Natl. Acad. Sci. USA 106 (2009), 18485-18490.

[7] S.S. Eaton, K.M. More, B.M. Sawant and G.R. Eaton, J. Am. Chem. Soc. 105 (1983), 6560-6567.

[8] M. Grote, E. Bordignon, Y. Polyhach, G. Jeschke, H.J. Steinhoff and E. Schneider, Biophys. J. 95 (2008), 2924-2938.

[9] K. Herz, A. Rimon, G. Jeschke and E. Padan, J. Biol. Chem. 284 (2009), 6337-6347.

[10] D. Hilger, Y. Polyhach, H. Jung and G. Jeschke, Biophys. J. 96 (2009), 217-225.

[11] D. Hilger, Y. Polyhach, E. Padan, H. Jung and G. Jeschke, Biophys. J. 93 (2007), 3675-3683.

[12] W.L. Hubbell, D.S. Cafiso and C. Altenbach, Nat. Struct. Biol. 7 (2000), 735-739.

[13] G. Jeschke and Y. Polyhach, Phys. Chem. Chem. Phys. 9 (2007), 1895-1910.

[14] G.G. Krivov, M.V. Shapovalov and R.L. Dunbrack Jr., Proteins 77 (2009), 778-795. 
[15] A. Pautsch and G.E. Schulz, J. Mol. Biol. 298 (2000), 273-282.

[16] K. Sale, L. Song, Y.-S. Liu, E. Perozo and P. Fajer, J. Am. Chem. Soc. 127 (2005), 9334-9335.

[17] O. Schiemann and T.F. Prisner, Quater. Rev. Biophys. 40 (2007), 1-53.

[18] D. Sezer, J.H. Freed and B. Roux, J. Phys. Chem. B 112 (2008), 5755-5767.

[19] F. Tombolato, A. Ferrarini and J.H. Freed, J. Phys. Chem. B 110 (2006), 26248-26259.

[20] Y.D. Tsvetkov, A.D. Milov and A.G. Maryasov, Usp. Khim. 77 (2008), 515-550.

[21] A. Volkov, C. Dockter, T. Bund, H. Paulsen and G. Jeschke, Biophys. J. 96 (2009), 1124-1141.

[22] A. Yamashita, S.K. Singh, T. Kawate, Y. Jin and E. Gouaux, Nature 437 (2005), 215-223. 


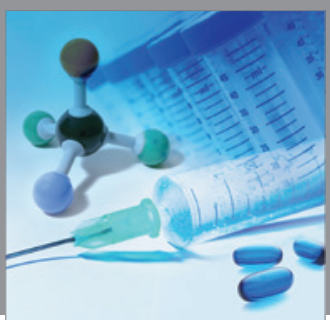

International Journal of

Medicinal Chemistry

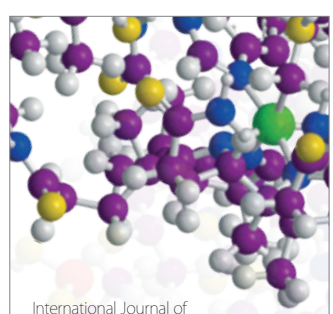

Carbohydrate Chemistry

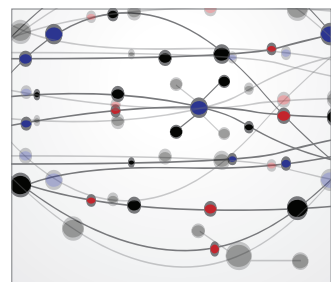

The Scientific World Journal
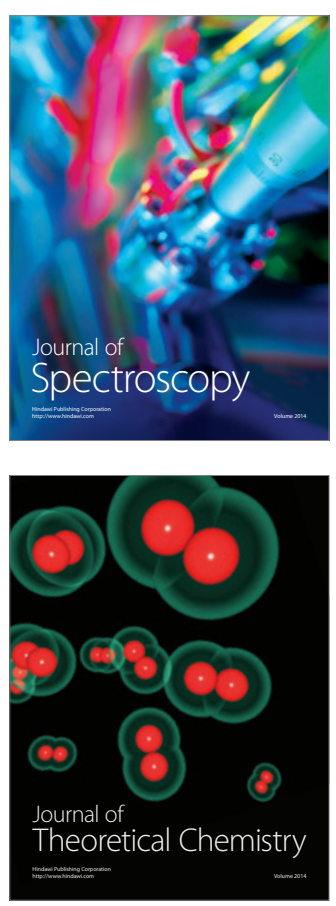
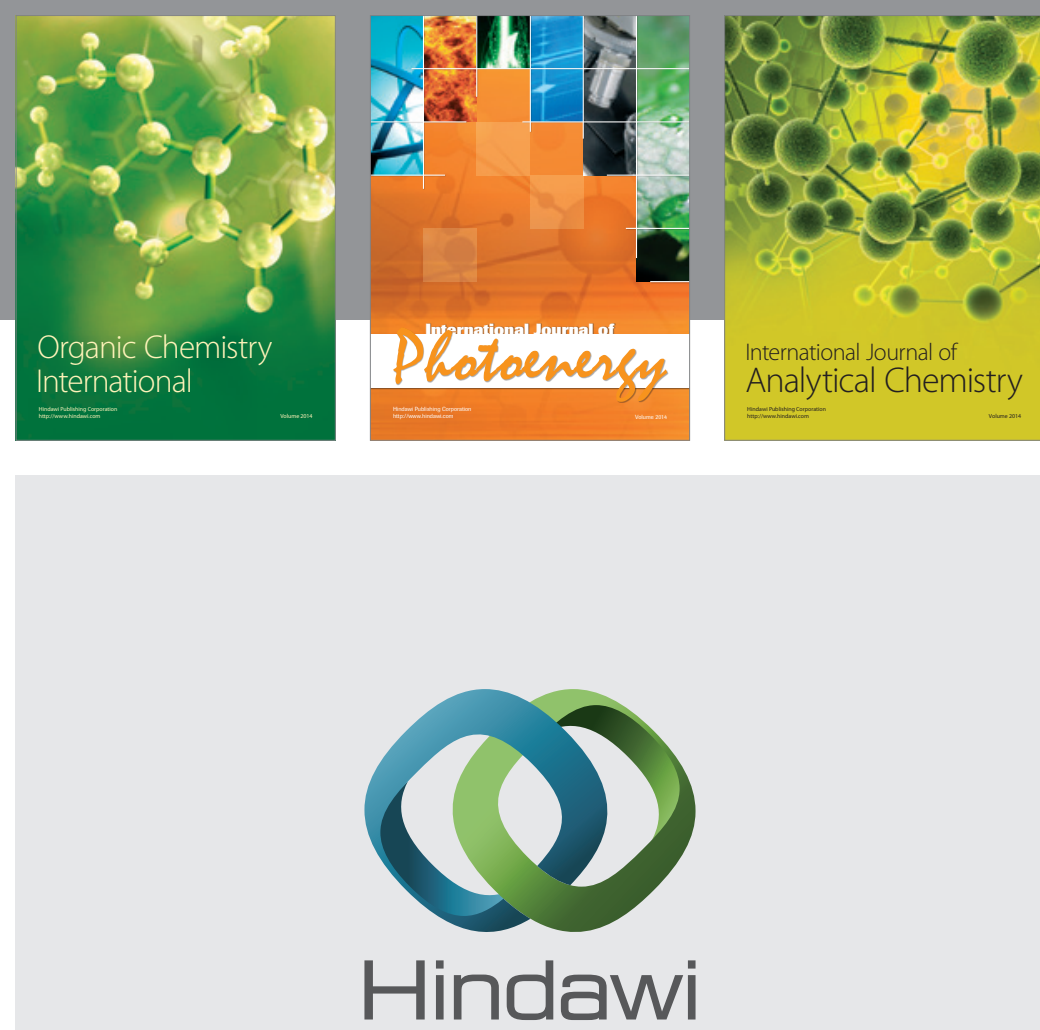

Submit your manuscripts at

http://www.hindawi.com
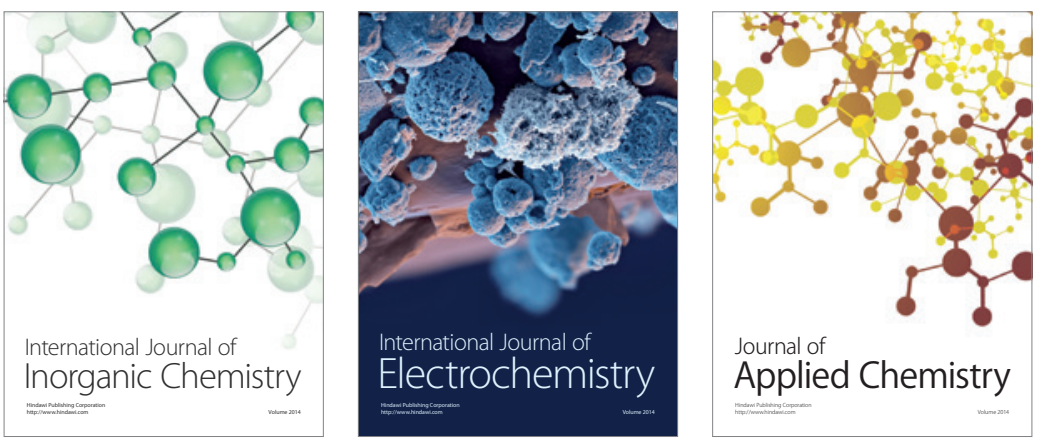

Journal of

Applied Chemistry
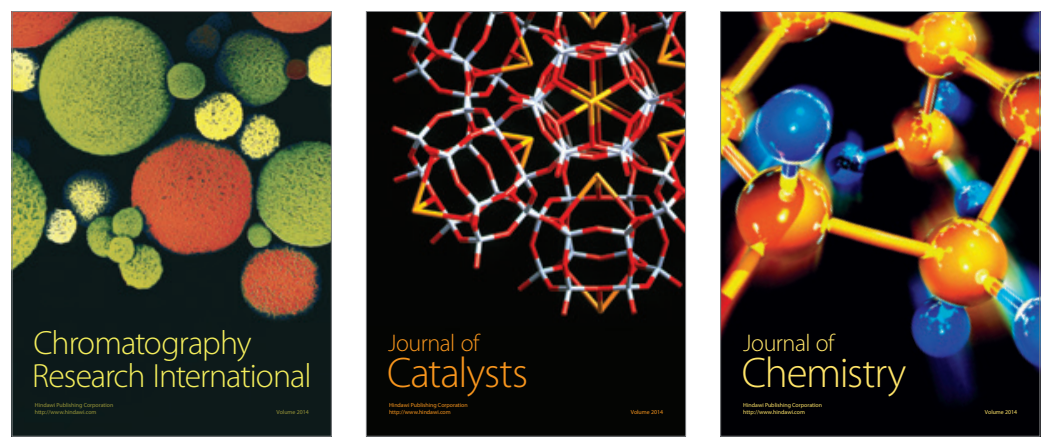
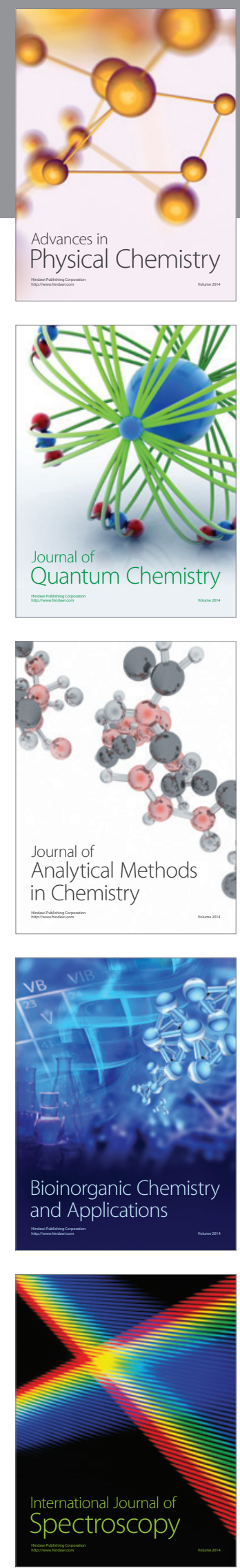